\title{
Refractive Index Shaping: In Vivo Optimization of an Implanted Intraocular Lens (IOL)
}

\author{
Ruth Sahler and Josef F. Bille
}

\subsection{Introduction}

Patient satisfaction is a constant pursuit in cataract surgery. In order to enhance the chance of each patient's postoperative satisfaction, cataract surgeons measure the refraction of the eye preoperatively and attempt to select the appropriate IOL for the patient, based on not only those measurements but also on the patient's needs and expectations. The overall number of implanted premium IOLs is still small compared to the overall number of implanted IOLs. Some surgeons avoid implanting premium IOLs because of their cost, possible limitations or side effects and therefore the possibility of an up unhappy patient [1].

A multifocal or toric IOL is more sensitive to decentration or tilt compared to a standard IOL. Postoperatively the IOL will settle in place and during this process the lens can still move. Current adjustable technologies only allow the adjustment of an implanted IOL if that IOL model was selected prior to cataract surgery and therefore prior to possible complications. After the first, desired adjustment is finalized the IOL is locked in and the IOL is no longer adjustable [2]. For standard, hydrophobic and hydrophilic IOLs

R. Sahler $(\bowtie)$

Perfect Lens LLC, Irvine, CA, USA

J. F. Bille

University of Heidelberg, Heidelberg, Germany the options for an undesired refractive outcome are ranging from spectacles, refractive surgery to lens explantation.

Studies suggest that a significant number of patients will require spectacle prescriptions after cataract surgery. For example, a clinical study found that $37.8 \%$ of cataract patients had preoperative astigmatism of more than $1.00 \mathrm{D}$ [3]. Furthermore, it was reported that postoperative astigmatism of greater than $0.75 \mathrm{D}$ has an adverse effect on the performance of a monofocal IOL [4]. Further, about $25.7 \%$ of patients who undergo conventional phacoemulsification and about $28 \%$ who undergo laser-assisted cataract surgery have a postoperative spherical error of more than $0.50 \mathrm{D}$, which is enough to adversely affect their distance vision [5].

Additionally, cataract surgery is generally performed in the elderly population, so most patients who do not choose multifocal IOL implantation will require reading correction postoperatively. Market Scope estimates that more than $90 \%$ of post-cataract patients are presbyopic. Taken together, all of these factors indicate that more than $50 \%$ of patients would benefit from a distance correction after cataract surgery, and another $40 \%$ might take advantage of multifocal optics.

Unfortunately, current premium IOLs cannot reliably solve these problems because there is a possibility for the IOL to move postoperatively. Further, the effects of wound healing are difficult to predict and add an additional complication. 
The refractive index shaping (RIS) technology in development by Perfect Lens, which can theoretically alter an IOL after it has been implanted and has settled in the eye. Preclinical studies have shown that a short $(<30 \mathrm{~s})$ in-office procedure can adjust acrylic IOL materials such that spherical, toric, and multifocal issues can be resolved permanently. The use of the femtosecond laser to create refractive index change in various materials has been studied for years. Ohmachi and Igo [6] showed a refractive index change of 0.056 in glass using a femtosecond laser. Ding et al. [7] used a femtosecond laser to obtain a refractive index change of up to 0.06 in hydrogel polymers.

Different theories regarding femtosecond laser material interactions which affect the refractive index change have been presented. The Rochester Group hypothesized that the light from the femtosecond laser induced crosslinking within a hydrophilic material and thus created an increase in the refractive index [8]. Takeshima et al. [9] believed the refractive index change in glass was caused by local heat effects from phase separation, while Katayama and Horiike [10] proposed that all changes resulted from either: (1) crosslinking, (2) phase separation, or (3) decomposition.
Recently a new process was discovered wherein existing molecules within a polymeric material become hydrophilic inside an intraocular lens (IOL) [11]. This change in hydrophilicity occurs when the polymeric material is immersed in an aqueous medium, while it is exposed to femtosecond laser radiation. The aqueous medium and the femtosecond laser radiation provide the chemical basis for the hydrophilicitybased refractive index change. After the exposure of the polymeric material to femtosecond laser radiation, water slowly diffuses to the sites with increased hydrophilicity forming hydrogen bonds, typically over a $24-72 \mathrm{~h}$ period of time, to create a refractive index change in the polymeric material.

\subsection{Technology Background}

\subsubsection{Femtosecond Laser-Induced Refractive Index Change (RIS)}

A new method for modifying the refractive index of polymeric materials has been developed, called Refractive Index Shaping (RIS) (Fig. 15.1a) [11]. a

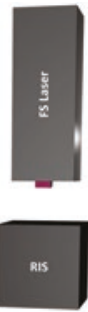

d
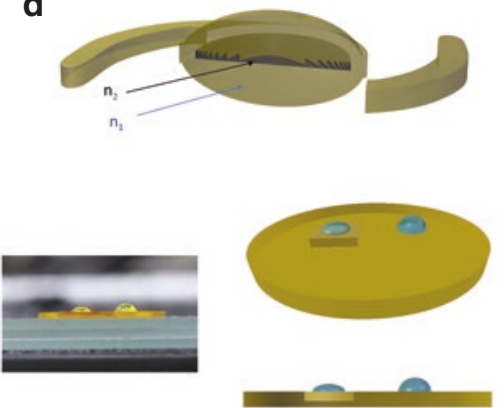

b

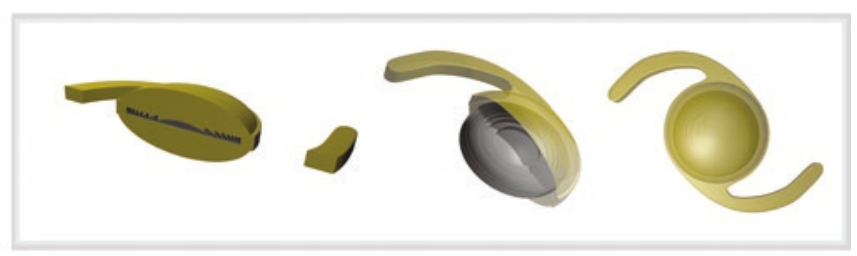

C

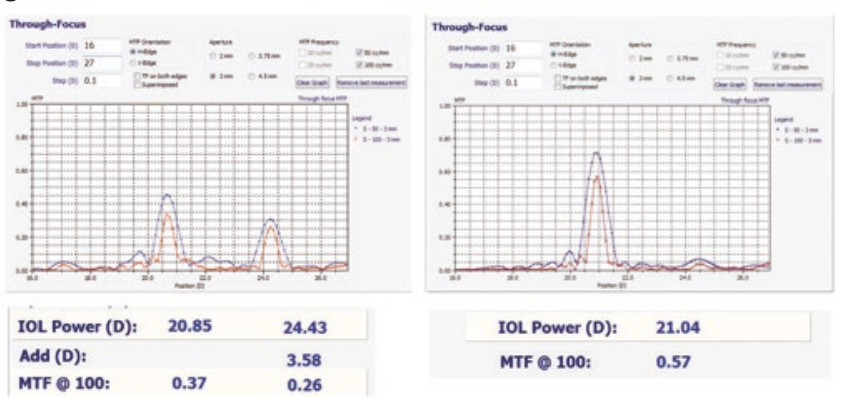

Fig.15.1 (a) Refractive Index Shaping (RIS), Femtosecond (FS) laser, refractive index of IOL $\left(\mathrm{n}_{1}\right)$ and refractive index of RIS lens $\left(\mathrm{n}_{2}\right)$. (b) Phase Wrapping. (c) Multifocal IOL to
Monofocal, before (left) and after (right) RIS-modification. (d) Hydrophilicity based $\Delta \mathrm{n}$ change (adapted with permission from Ref. 12, The Optical Society) 
High repetition rate femtosecond laser pulses are directed to a designated area to create a "lens" inside an IOL. RIS changes the refractive characteristics of the polymeric material without cutting the material. The RIS-lens is a gradient lens, with the related refractive index change generated by the instantaneous energy of the laser pulse, which is regulated by an acousto-optical modulator (AOM) at approx. $1 \mathrm{MHz}$ speed. The physical parameters of the procedure, like scan speed, wavelength, pulse rate, energy per pulse, etc., are provided in [13], as well as data on the homogeneity of refractive index change. In preparation of a RIS lens, the femtosecond laser is directed to a small area within the polymeric IOL. The laser light has several effects on the acrylic material: (1) the most recognized is that the laser light heats the material and causes a change in the material as a result of the heat, and (2) if the proper wavelength is utilized, the exposure to the laser light will alter the polarity of certain molecules within the polymeric material and change the hydrophilicity of the polymeric material. The change in hydrophilicity drives a large, repeatable and homogeneous change in refractive characteristics, which does not depend on the accumulation of heat and therefore can be used with a fast scan speed, allowing for in vivo application.

\subsubsection{Phase Wrapping}

In a traditional convex lens, one would be limited to a height of $200 \mu \mathrm{m}$ (central slab area) in order to adjust the optical power of the IOL. The power for a $6 \mathrm{~mm}$ lens with a height of $200 \mu \mathrm{m}$ would be $0.44 \mathrm{dpt}(\Delta \mathrm{n}=0.01)$. Phase wrapping is a process which is used to create a RIS "lens" with enhanced diopter change, without increasing the height of the "lens". Thus, a convex lens is reduced to a thin layer of approx. $50 \mu \mathrm{m}$ thicknesses, creating multiple refractive zones. The different phase levels are created by controlling the energy per pulse and focal spot. For a "lens" with a diameter of $6 \mathrm{~mm}$, one zone corresponds to 0.1 diopter (Fig. 15.1b).

\subsubsection{Example of RIS-Procedure: Change of Diffractive Hydrophilic IOL into a Monofocal IOL}

The possibility of changing a diffractive multifocal IOL into a monofocal IOL was evaluated. A suitable lens design was created to match the diffractive power and energy split of the diffractive multifocal IOL, as depicted in Fig. 15.1c. The original IOL measured $20.85 \mathrm{D}$ with an add power of $3.58 \mathrm{D}$ and a modulation transfer function (MTF) of 0.37 and 0.26 . After RIS shaping, the IOL measured as a monofocal IOL at $21.04 \mathrm{D}$ with an MTF of 0.57. The IOL shown in Fig. 15.1c, before (left), was a commercial diffractive multifocal IOL. The RIS-process was imposed to change the lens from multifocal to monofocal. The inverse process, i.e. creation of multifocality in a monofocal hydrophobic IOL is shown also in [14]. A RIS-lens can be 'erased', sub-sequentially, by e.g. creating a RIS-lens with opposite refraction in an adjacent layer.

\subsubsection{Hydrophilicity-Based $\Delta n$ Change}

To demonstrate that the hydrophilicity of the polymeric material has been changed, two areas of polymeric material were compared. One area of the material had not been treated, and the adjacent area was treated with the femtosecond laser. To test whether the treatment created a hydrophilic area, the wetting angle measurement technique was employed [15]. The treated and untreated sections of an acrylic hydrophobic material were exposed to a drop of water. Figure 15.1d (left) shows the drop of water on a treated area, while Fig. 15.1d (right) displays a water drop placed on an untreated area of the lens. The angle of the drop, on top of the treated material, in Fig. 15.1d (left), is $\sim 64^{\circ}$, which indicates that it is in contact with a hydrophilic surface. The angle of the drop, on top of the untreated material, in Fig. 15.1d (right), is $\sim 87^{\circ}$, which indicates that the drop is in contact with a hydrophobic surface. The change in hydrophilicity demonstrates that the treatment with a femtosecond laser created a hydrophilic area. 


\subsection{Microscope Study: Methods and Materials}

Two different microscope setups have been used for the study: Laser Induced Fluorescence (LIF) microscopy (Sect. 15.3.1) [16] and Raman microscopy (Sect. 15.3.2) [17]. Various hydrophilic and hydrophobic intraocular lens materials were studied (Sect. 15.3.3). Each microscope is being used to identify exactly what molecular changes occur upon exposure of the polymeric material to the light of the femtosecond laser.

\subsubsection{Laser-Induced Fluorescence (LIF) Microscopy, STED Contrast}

The STED (Stimulated Emission Depletion) microscope uses a low power pulsed supercontinuum laser source (WhiteLase SC450-PP-HE, Fianium, Southampton, UK) for excitation at virtually any optical wavelength. After removal of the IR part of the supercontinuum spectrum using a $760 \mathrm{~nm}$ short pass filter, the desired excitation wavelength is selected using an acousto-optical tunable filter (AOTF, PCAOM-VIS, Crystal Technologies, Palo Alto, USA). The beam passes the AOTF three times in order to suppress the undesired wavelength range of the supercontinuum spectrum; the triple pass suppresses 1000 times better than a regular single pass. The STED laser is a frequency-doubled pulsed fiber laser (Katana-08 HPKA/40/07750/600/1600/FS) providing $600 \mathrm{ps}$ pulses of up to $40 \mathrm{~nJ}$ pulse energy at a wavelength of $775 \mathrm{~nm}$. The STED laser can be triggered electronically over a wide frequency range $(25 / 40 \mathrm{MHz})$ which greatly simplifies the synchronization of the excitation and STED pulses. The STED laser is triggered by the pulsed supercontinuum laser operating at $38.6 \mathrm{MHz}$.

\subsubsection{Raman Microscopy}

Raman spectra were recorded on a commercial HORIBA XploRA PLUS Raman Microscope (HORIBA Jobin Yvon GmbH, Bensheim, Germany). All spectra were measured with a $10 \times$ objective with a $600 \mathrm{~g} / \mathrm{mm}$ grating. The wavelength of the continuous wave excitation laser source was $785 \mathrm{~nm}$ (with a laser output of approximately $100 \mathrm{~mW}$ ). Raman spectra were acquired both in the fingerprint $\left(200-1800 \mathrm{~cm}^{-1}\right)$ and highwavenumber (2400-3800 $\left.\mathrm{cm}^{-1}\right)$ regions.

\subsubsection{Materials}

The microscopic study was performed on three different IOL materials. The following samples were studied: (1) hydrophilic acrylic material without yellow dye [18]: (1a) Hydrophilic acrylic intraocular lens (see e.g. Sect. 15.4.2.1) and (1b) Hydrophilic acrylic strip, cut from a hydrophilic acrylic button (see e.g. Sect. 15.4.2.2); (2) hydrophobic acrylic strip with yellow dye (blue blocking), cut from a hydrophobic acrylic button [19], and (3) hydrophobic acrylic strip without yellow dye, cut from a hydrophobic acrylic button [20]. The hydrophilic acrylic intraocular lens had a refractive power of five diopters, the strips were cubes of approximately $10 \mathrm{~mm} \times 2 \mathrm{~mm} \times 2 \mathrm{~mm}$ dimension and exhibited no refractive power. All strips are made from buttons, with the material specified in [18-20]. The acrylic buttons were disc shaped, $10 \mathrm{~mm}$ in diameter and $2 \mathrm{~mm}$ thick.

The chosen microscopic techniques provide information on the chemical nature of the process, on the electronic (fluorescence) as well as the molecular (Raman) level. CARS-microscopy is sensitive to refractive index changes, due to the four-wave mixing feature. In case of the clear hydrophilic acrylic material, LIF microscopy, STED microscopy and Raman microscopy were applied. The yellow hydrophobic material as well as the clear hydrophobic material was studied with LIF microscopy and STED microscopy.

\subsection{Chemical Basis for RIS}

\subsubsection{Enhancement of Hydrophilicity by Femtosecond Laser Excitation}

In Fig. 15.2, the photo-induced hydrolysis of polymeric material in aqueous media is presented, 
in which two hydrophilic functional groups, acid group and alcohol group, are produced [12]. This result is similar to the results found in previous research on surface treatment of PMMA, with femtosecond laser two-photon excitation [21], and excimer laser UV excitation [22].

Another possible mechanism for enhancement of hydrophilicity is two-photon depolymerization [23]. Zhou et al. [24] use a random copolymer of tetrahydropyranyl metacrylate (THPMA) and methyl methacrylate (MMA) polymer doped with BSB-S ${ }_{2}$ as the UV photoacid generators (PAG) for microfabrication. At the laser focal spot, the THPMA groups were converted to carboxylic acid groups due to photo-generated acid-induced ester cleavage reactions, and were therefore rendered soluble in aqueous base developer. This process may

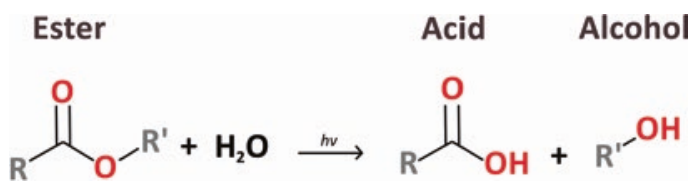

Fig. 15.2 Photo-induced hydrolysis (adapted with permission from Ref. 12, The Optical Society) essentially contribute to the increase of hydrophilicity in laser treated areas in hydrophobic lens materials.

\subsubsection{Femtosecond Laser Excited Fluorescence in a Hydrophilic Intraocular Lens}

\subsubsection{Section of a Hydrophilic Intraocular Lens}

The schematic sketch of the hydrophilic intraocular lens of five diopters is shown in Fig. 15.3a. The material is a clear material and measures $6 \mathrm{~mm}$ in diameter, and the treated area is within a $4 \mathrm{~mm}$ circle in the center of the lens. As shown in Fig. 15.3b, the newly formed hydrophilic molecules in the laser-treated area can be imaged by Laser Induced Fluorescence ("LIF") microscopy, visualizing the phasewrapped RIS-lens by green fluorescent light emission, with blue excitation and wide field illumination (10x objective). Different shades of green correspond to different amounts of fluorescence light, indicating different amounts of newly formed hydrophilic polar molecules.

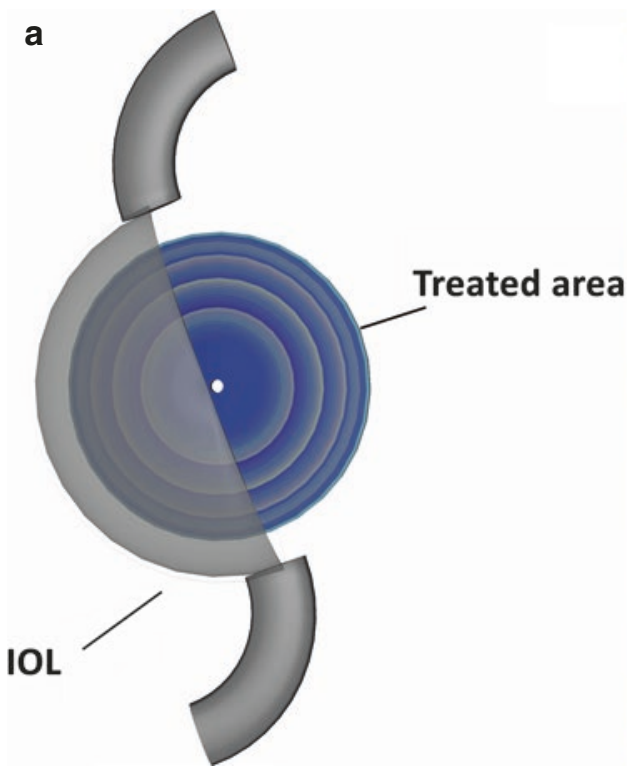

Fig. 15.3 (a) Schematic sketch of hydrophilic acrylic lens (five diopters), RIS-treated area $4 \mathrm{~mm}$ circle in the center of the intraocular lens. (b) Fluorescence image of a

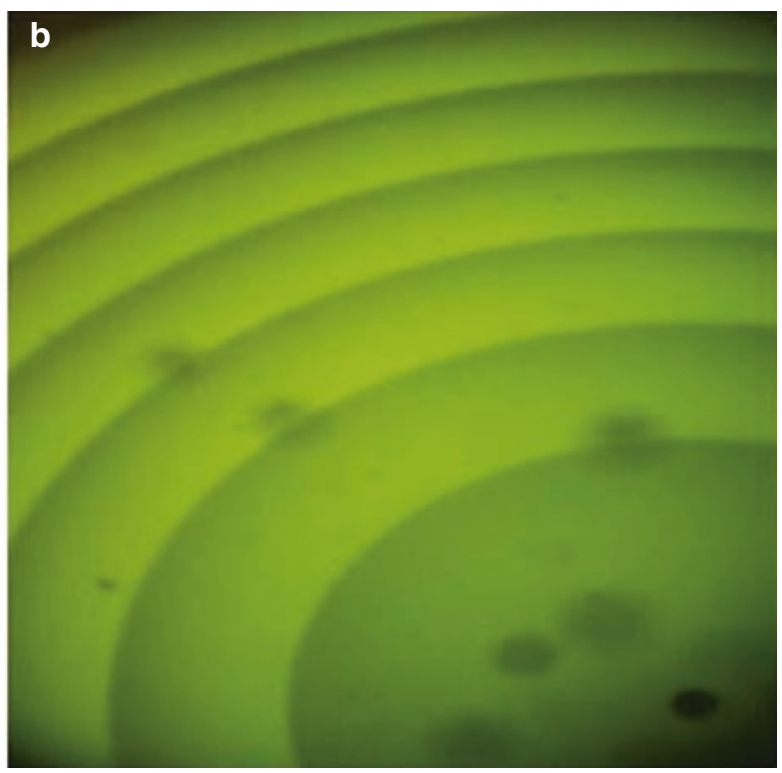

RIS-lens, inscribed in the hydrophilic acrylic lens, sketched in this figure (a) (adapted with permission from Ref. 12, The Optical Society) 
The fluorescence image reflects the homogeneity and repeatability of refractive index change in the laser treated areas.

\subsubsection{Fluorescent Light, Originating From Newly Created Fluorophores (Simultaneous Scans)}

In Fig. 15.4, the simultaneous scanning of a laser excited area with light of two different wavelengths, e.g. $600 \mathrm{~nm}$ (Left image, fluorescence detection at $628 \mathrm{~nm}$ ) and $650 \mathrm{~nm}$ (Right image, fluorescence detection at $708 \mathrm{~nm}$ ) is depicted, demonstrating the detection of spatially distributed fluorophores in "On/Off" states. When the fluorophore has exposure to light of the correct wavelength it absorbs energy and creates fluorescent light. This so-called "Blinking" indicates the presence of single fluorophores, with active or silent behavior. In the upper middle part, the two instantaneous images are overlaid, labeling the left image in red color and the right image in green color. Note the scale bar of $1 \mu \mathrm{m}$, demonstrating submicron resolution of the images. The regions imaged in Fig. 15.4 are only approx. $10 \mu \mathrm{m}$ in size, and are selected in fully treated areas, resulting in homogeneous appearances.

\subsubsection{Femtosecond Laser Excited Fluorescence in a Hydrophobic Intraocular Lens}

In Fig.15.5, various RIS lenses, written in clear hydrophobic lens material [20] are imaged with fluorescence microscopy (Cylindrical RIS lens (Fig. 15.5a), Spherical RIS lens (Fig. 15.5b), Sperocylindrical RIS lens (Fig. 15.5c).

In Fig. 15.6a and b, transmission (top) and fluorescence (bottom) images of a hydrophobic strip are depicted [20]. A RIS lens was patterned (Fig. 15.6a and b, arrows) in the center of the hydrophobic strip.

In Fig. 15.6c, fluorescence spectra from the RIS-pattern of clear hydrophobic mate-

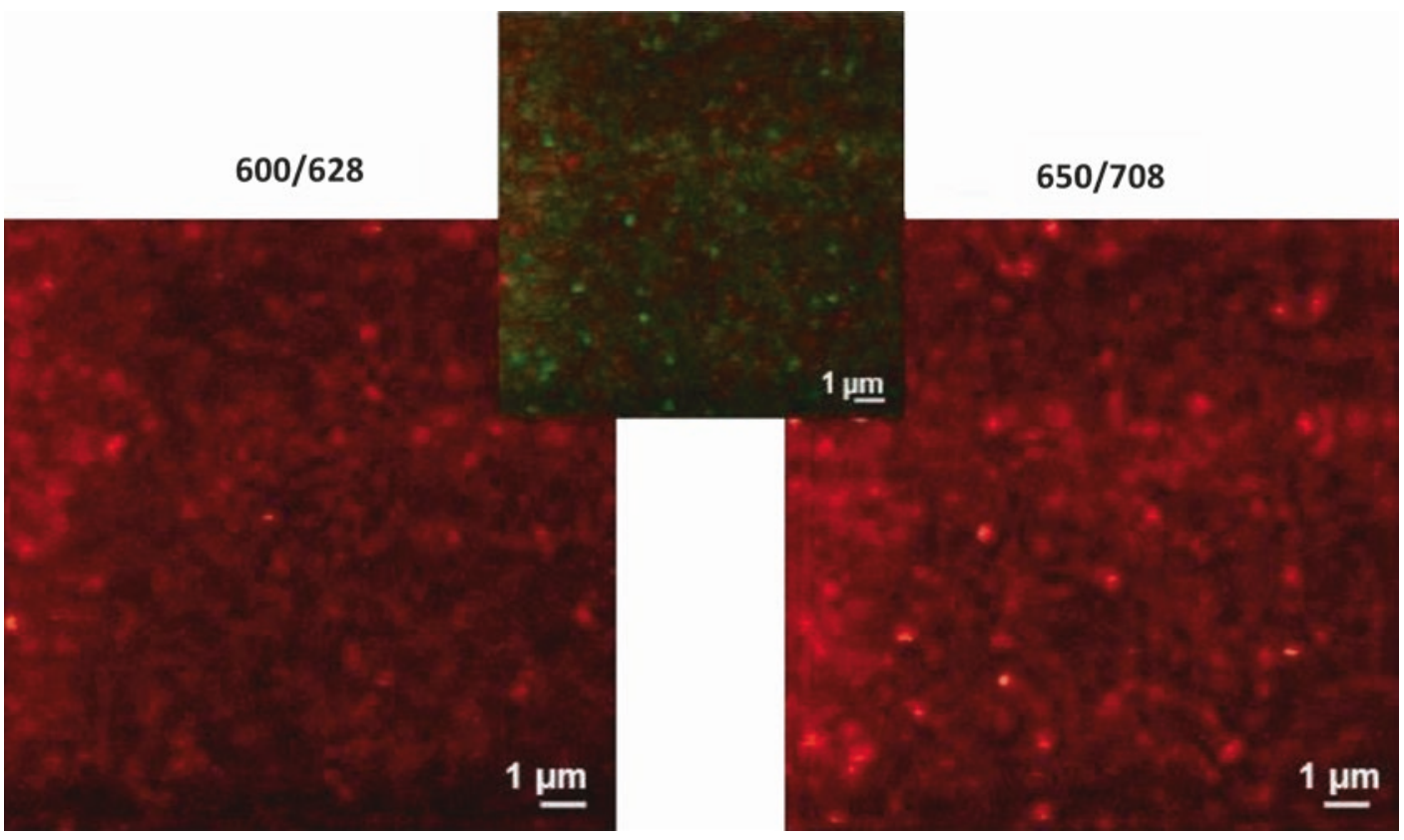

Fig. 15.4 Simultaneous scans at 600 and $650 \mathrm{~nm}$. Left image-fluorescence detection at $628 \mathrm{~nm}$, right imagefluorescence detection at $708 \mathrm{~nm}$. In the upper middle part, two instantaneous images were overlaid, labeling the left image in red color and right image in green color. The imaged regions were approx. $10 \mu \mathrm{m}$ in size, and were selected in fully treated areas, resulting in homogeneous appearances (adapted with permission from Ref. 12, The Optical Society) 


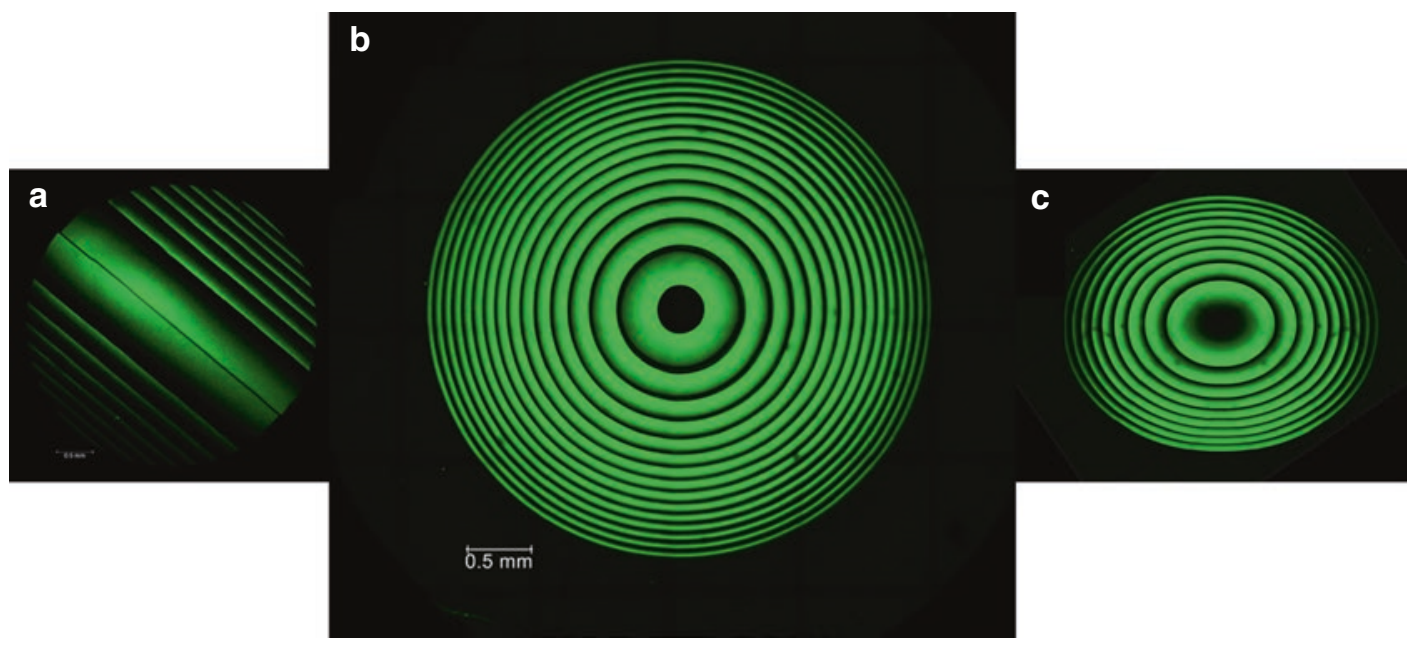

Fig. 15.5 Fluorescence images of hydrophobic, RIS lenses, (a) cylindrical, (b) spherical and (c) spherocylindrical RIS lens

rial [20] are shown, with excitation/emission at $405 / 500 \mathrm{~nm}$, and 488/535 nm, respectively. The spectra closely resemble the spectra of the RIS pattern of yellow hydrophobic material [19], as well as the spectra from the hydrophilic material [18], reaffirming the fact that similar fluorescent molecules are generated in hydrophilic and hydrophobic materials.

Figure 15.6d (left) displays simultaneous $\mathrm{xz}$-scans at three excitation wavelengths (exc $470 \mathrm{~nm}$, em 525/50 nm (upper left); exc $605 \mathrm{~nm}$, em 628/32 nm (upper right); exc $650 \mathrm{~nm}$, em $708 / 75 \mathrm{~nm}$ (lower left)). The bright spot marks the surface of the clear hydrophobic material.

The fluorescence appeared strongest at $605 \mathrm{~nm}$ excitation while it was very weak at blue light excitation. Inside the bulk material the intensity drops after a few microns. This is probably caused by a mismatch of the refractive index between the immersion oil and the bulk material. The lower narrow line marks the coverslip glass surface on top of which the sample was mounted.

The clear hydrophobic material was imaged at two fluorescence bands simultaneously (see Fig. 15.6d (right)) (exc $605 \mathrm{~nm}$, em 628/32 nm (upper left) and exc $650 \mathrm{~nm}$, em 708/75 nm (upper right). The fluorescence emissions appear homogeneous in both wavelength bands at a dif- fraction limited resolution level of $230 \mathrm{~nm}$. The regions imaged in Fig. 15.6d are only approx. $10 \mu \mathrm{m}$ in size, and are selected in fully treated areas, resulting in homogeneous appearances.

The fluorescent molecules in the clear hydrophobic material bleach [20], i.e. photo-convert into a non-fluorescent species, upon excitation, similar to common organic fluorescent molecules. Figure 15.6e (left) shows a darker square region in the center, which was previously scanned several times. The regions imaged in Fig. 15.6d and e are only approx. $10 \mu \mathrm{m}$ in size, and are selected in fully treated areas, resulting in homogeneous appearances.

The fluorescent species in the clear hydrophobic material can be stimulated from the excited to the ground state similar to common organic fluorescent molecules. Figure 15.6e (right) shows the fluorescence intensity measured in a STED microscope (see [16]), with excitation laser and STED laser simultaneously switched on. The brighter band shows a region where the STED laser was temporarily switched off. No finer structures could be found with STED imaging contrast. The noise is shot noise from a photon count per pixel of 17 in the bright region and a count of 6 in the regions where the STED laser was on. 
a
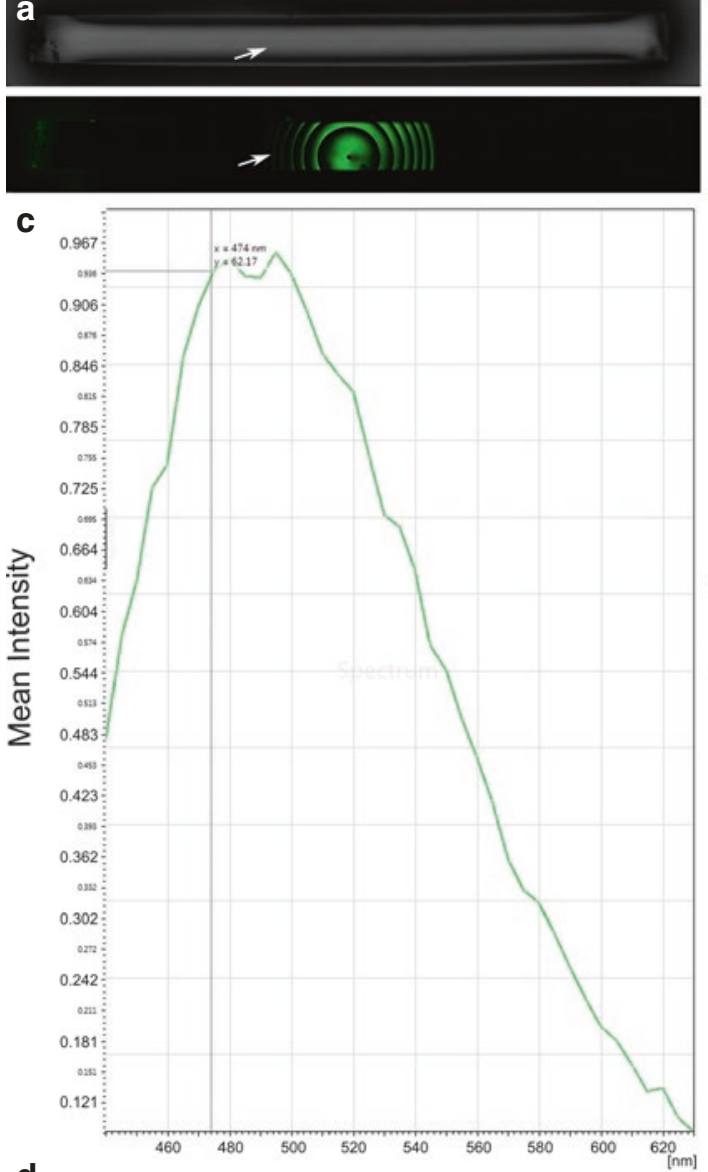

d

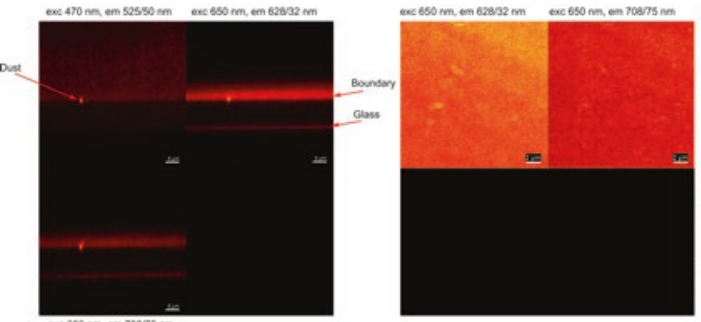

$x z$ - section clear hydrophobic stripe
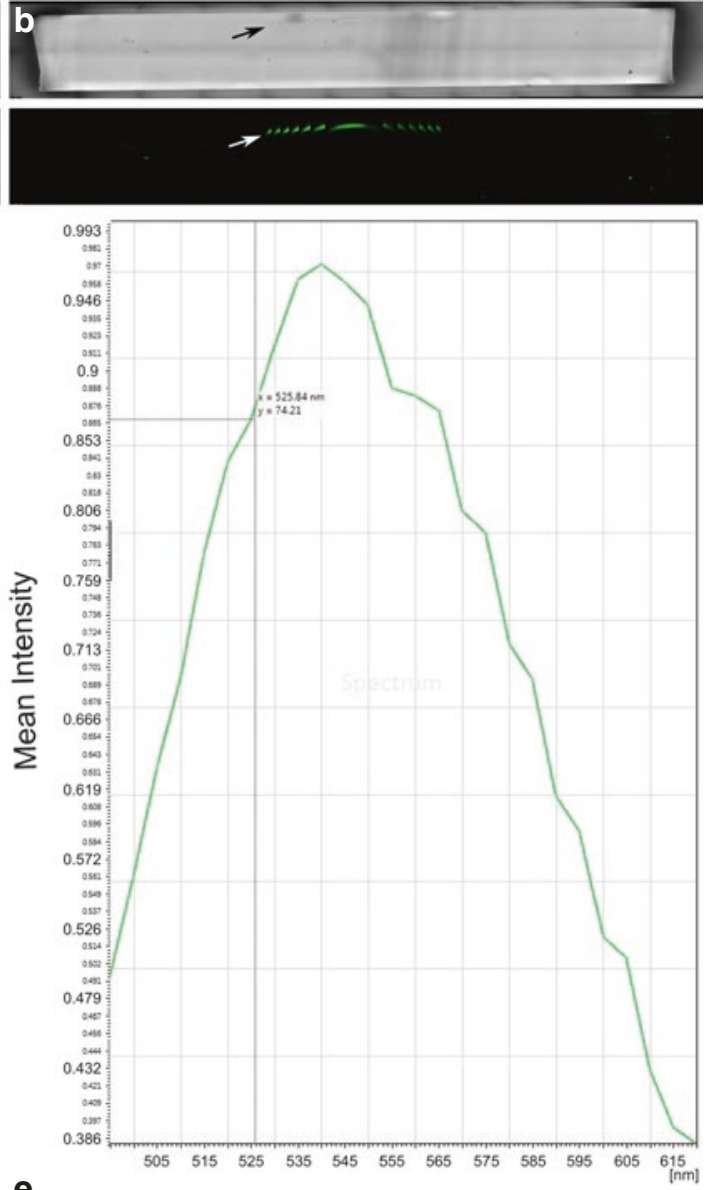

e
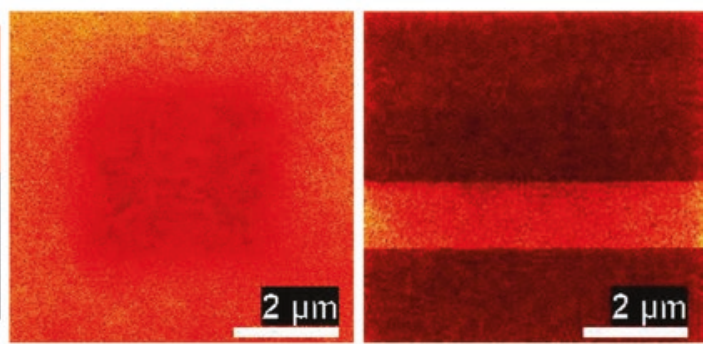

exc $605 \mathrm{~nm}$, em $628332 \mathrm{~nm}$, STED $775 \mathrm{~nm}$
Fig. 15.6 (a) Hydrophobic clear strip (birdview): transmission image (top), fluorescence image (bottom) and the RIS patterns indicated by arrows. (b) Hydrophobic clear strip (sideview): transmission image (top), fluorescence image (bottom). (c) Fluorescence spectra, excitation at $405 \mathrm{~nm}$ and emission max. at $500 \mathrm{~nm}$ (top), excitation at $488 \mathrm{~nm}$ and emission max. at $535 \mathrm{~nm}$ (bottom) (sample: Clear hydrophobic strip [20]). (d) Left: Magnified a few $\mu \mathrm{m}$ sized confocal xzslice (side view) across a bright part of the Fresnel pattern. Right: Magnified confocal xy-slice (top view, at the samples surface) at a bright part of the Fresnel pat- tern. The fluorescence images were taken simultaneously at $470 \mathrm{~nm}$, resp. $605 \mathrm{~nm}$, resp. $650 \mathrm{~nm}$ excitation. (e) High resolution fluorescence xy-images (top view) of clear hydrophobic strip. Left: The darker squared field shows an area which was previously scanned and gradually bleached. Right: The bright band indicates an area where the STED beam was switched off temporarily while the full image was scanned. Thus, the newly created fluorophores show analogous behavior (bleaching and stimulated emission) like regular fluorescent dyes (adapted with permission from Ref. 12, The Optical Society) 


\subsubsection{Identification of Fluorescent Molecules as Benzenamines}

The excitation/emission spectra of a laser excited area are plotted in a three dimensional graph, with the excitation wavelengths on the abscissa and the emission wavelengths on the ordinate (see Fig. 15.7a). The z-axis is depicting the intensity of the fluorescence light, emitted by the fluorophores. The fluorescence excitation and emission scan was done with a TCS SP8 $\mathrm{X}$ system (Leica Microsystems, Mannheim). Data analysis and the graphs were generated using the Leica confocal software LASX. The microscope was equipped with a white light laser. The highest fluorescence light emission was generated at a wavelength of $470 \mathrm{~nm}$ of the white light laser. The corresponding emission spectrum extends over a broad spectral region, from 500 to $650 \mathrm{~nm}$, indicating the formation of hydrophilic polar molecules. This graph demonstrates the sensitivity of the polymer molecules to laser light excitation.

With an excitation wavelength of $472 \mathrm{~nm}$, the emission spectrum of the fluorophore is centered at $527 \mathrm{~nm}$, as depicted in the lower left of Fig. 15.7b (TCS SP8 X (Leica Microsystems $\mathrm{GmbH}$ )). In the upper left of Fig. 15.7b, a typical excitation/ emission spectrum of an aromatic carboxylic acid Rhodamine Green Carboxylic Acid is plotted for comparison, with excitation at $480 \mathrm{~nm}$ and emission centered at $525 \mathrm{~nm}$. Thus, the spectral signature of the femtosecond laser generated polar molecule is similar to the characteristics of an aromatic carboxylic acid. Based on the chemical composition of the acrylic material with UV-dopant copolymer, the spectral signature of the femtosecond laser generated polar molecules points to the class of benzenamines, like N-phenyl-4-(phenylazo)benzenamine (C18H15N3). For comparison, the excitation/emission spectra of a pure acrylic material, e.g. PMMA, are shown on the lower right side, which are positioned in the deep UV, indicating that the UV-absorber molecules, which get excited by two-photon absorption, are essential to initiate the observed molecular changes.

\subsubsection{Raman Spectra of Hydrophilic Material}

In Fig. 15.8, Raman spectra are depicted which were recorded at three different positions of the hydrophilic material: Left (RIS-pattern, blue), Right (RIS-pattern, red), Center (Untreated area, black). The high wavenumber (2400$3800 \mathrm{~cm}^{-1}$ ) region of the Raman spectra shown in Fig. 15.8a is dominated by two features. The sharp feature in the region $2800-3000 \mathrm{~cm}^{-1}$, which is composed of three distinct vibrational bands, can be assigned to stretching vibrations of $\mathrm{CH}$, and $\mathrm{CH} 2$ functional groups [25]. The relatively broad feature ranging from $3100 \mathrm{~cm}^{-1}$ a

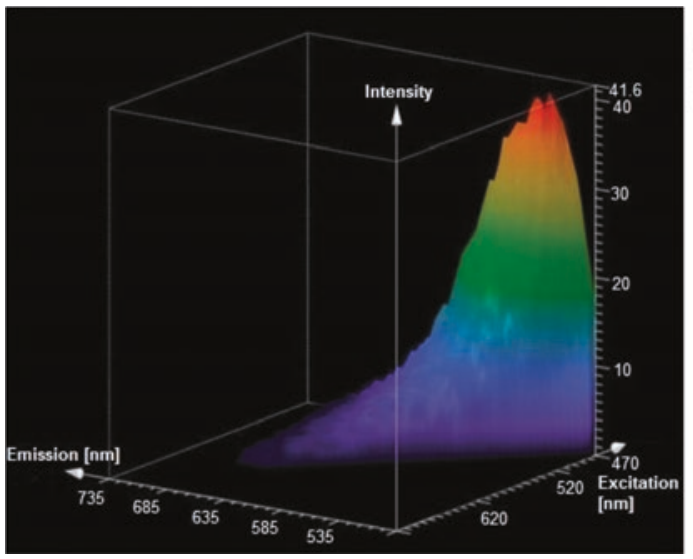

b

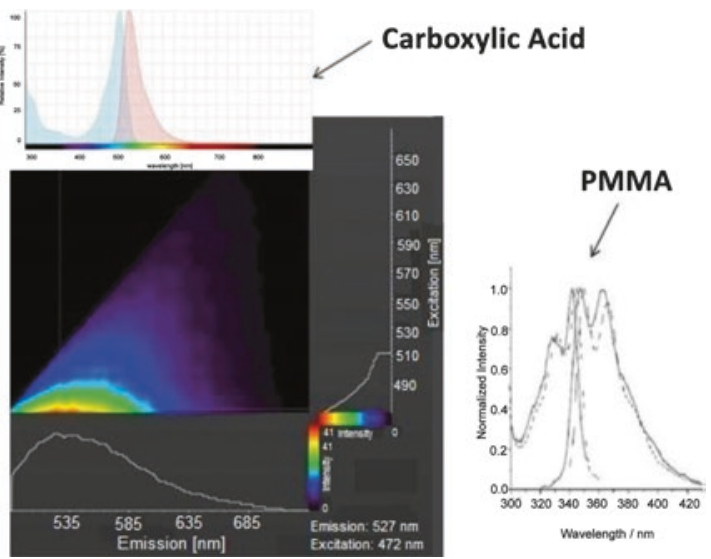

Fig. 15.7 (a) Excitation/Emission Spectra of fluorescent molecule. (b) Identification of fluorescent molecule (adapted with permission from Ref. 12, The Optical Society) 


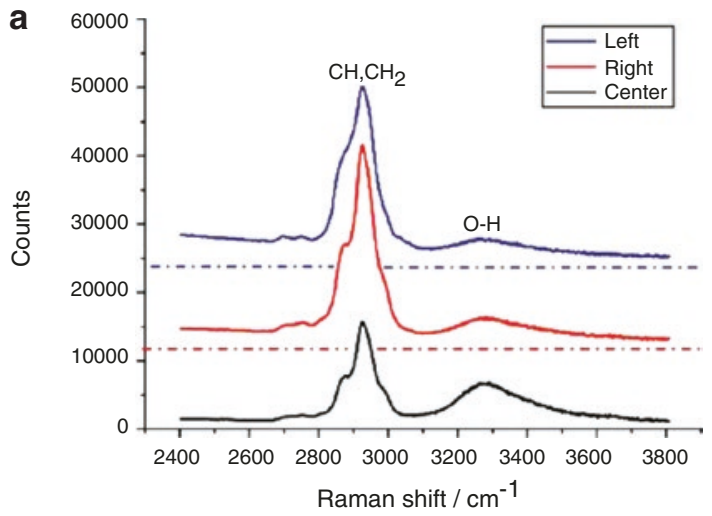

Fig. 15.8 Raman spectra of a hydrophilic material: (a) High-frequency part, (b) Low-frequency part. Dashed dotted horizontal lines represent the zero signal base lines

up to ca. $3600 \mathrm{~cm}^{-1}$ with a frequency maximum around $3300 \mathrm{~cm}^{-1}$ is characteristic for stretching vibrations of hydrogen bonded $\mathrm{OH}$ groups of water molecules in the hydrophilic polymer material [26]. The assignments of several distinct spectral features in the fingerprint region (200-1800 $\mathrm{cm}^{-1}$ ), which are assigned in the Raman spectra of Fig. 15.8b, indicate that the base material of the hydrophilic strip largely resembles the molecular structure of a poly2-hydroxyethylmethacrylate (PHEMA) polymer $[25,27]$. In the latter case the capability for the high water uptake of the material can be attributed to the presence of $\mathrm{OH}$ groups along the flexible polymer backbone, which can form primary hydrogen bonds with water molecules.

As can be seen in Fig. 15.8a the overall $\mathrm{OH}$ band intensity is significantly diminished in the Raman spectra measured in the lasertreated areas (Left and Right) as compared to the untreated area (Center) of the strip. This is consistent with consumption of $\mathrm{H} 2 \mathrm{O}$ molecules in the laser-treated areas due to the photo-induced hydrolysis reaction shown in Fig. 15.2. Furthermore, the reduction of the $\mathrm{OH}$ band intensity in the laser-treated region is paralleled by a significant increase of the $\mathrm{CH}$ and $\mathrm{CH} 2$ stretching vibration band intensities, which further indicates reaction of the polymer material upon femtosecond laser treatment. The latter fact is confirmed by the observed signifi-

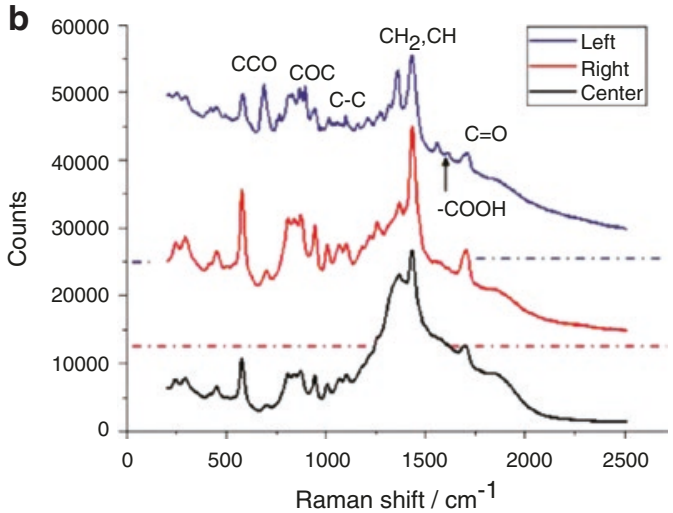

of the respective Raman spectra, which were shifted vertically for the sake of clarity (adapted with permission from Ref. 12, The Optical Society)

cant change of the low frequency range Raman spectra (Fig. 15.8b) upon laser treatment. The Raman spectra taken within the treated area (Right, Left in Fig. 15.8b) exhibit a noticeable contribution of background fluorescence light in the low frequency region $\left(200-2500 \mathrm{~cm}^{-1}\right)$, due to excitation/emission processes of newly created fluorophores. In contrast, there is almost no fluorescence background in the untreated area (Center in Fig. 15.8b), demonstrating, that fluorophores are solely generated by the irradiation with the femtosecond laser. Considering the possible presence of UV-blocker/stabilizers in the polymer material (such as e.g. benzotriazole derivatives $[28,29])$ the newly created fluorescent molecules might be phenazine derivatives, which could be formed by reaction sequence initiated by the femtosecond twophoton laser induced photochemical activation of the benzotriazole copolymer derivatives. Again these molecules remain in their existing place and are modified by the exposure to the laser light. Furthermore, a new molecular vibration in the region $1600-1620 \mathrm{~cm}^{-1}$ that is observed in the laser-treated area (Fig. 15.8b, Left) which can be assigned to an aryl carboxylic acid $\mathrm{COOH}$ moiety [30]. This entity is a residual of the original reaction initiated by the laser light. The laser generated fluorophores could be phenazine-1-carboxylic acid molecules (see Table 15.1). 
Table 15.1 Spectral band assignments

\begin{tabular}{l|l}
\hline $\begin{array}{l}\text { Frequency in } \\
\mathrm{cm}^{-1}\end{array}$ & Possible assignments \\
\hline $550-610$ & CCO stretch \\
\hline $890-900$ & COC stretch \\
\hline $1080-1120$ & C-C stretch \\
\hline $1340-1375$ & $\mathrm{CH}_{2}$ twist and rock \\
\hline $1400-1460$ & $\begin{array}{l}\mathrm{CH}_{2} \text { in-plane bending, } \mathrm{CH} \\
\text { deformation }\end{array}$ \\
\hline $1600-1620$ & COOH stretch \\
\hline $1650-1750$ & $\mathrm{C}=\mathrm{O}$ stretch \\
\hline $2800-3000$ & $\mathrm{C}-\mathrm{H}$ stretch of $\mathrm{CH}, \mathrm{CH}_{2}$ groups $)$ \\
\hline $3100-3600$ & $\mathrm{O}-\mathrm{H}$ stretch \\
\hline
\end{tabular}

\subsection{In Vivo Lens Shaping Proof of Concept}

\subsubsection{Concept and Repeatability}

In Fig. 15.9, the original proof of concept for a two diopter RIS lens within an IOL is depicted, with a starting diopter of 5.05D. The creation of the RIS lens altered the overall lens diopter to 2.91D. The pre-lens MTF was 0.53 for $100 \mathrm{lp} /$ $\mathrm{mm}$, the post-lens MTF was 0.40 for $100 \mathrm{lp} / \mathrm{mm}$. The shaping algorithm was further improved a

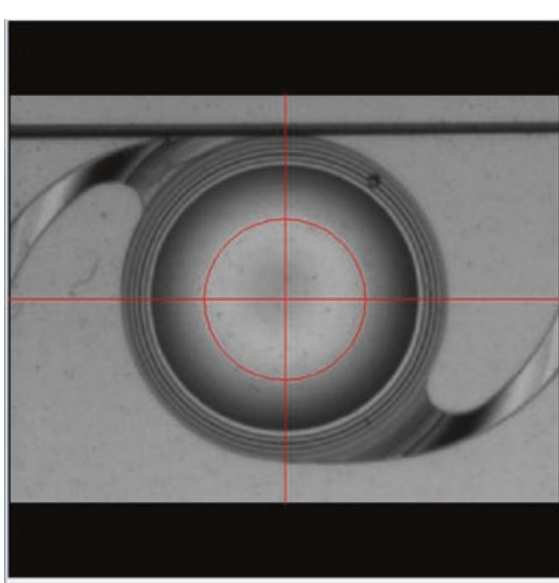

b

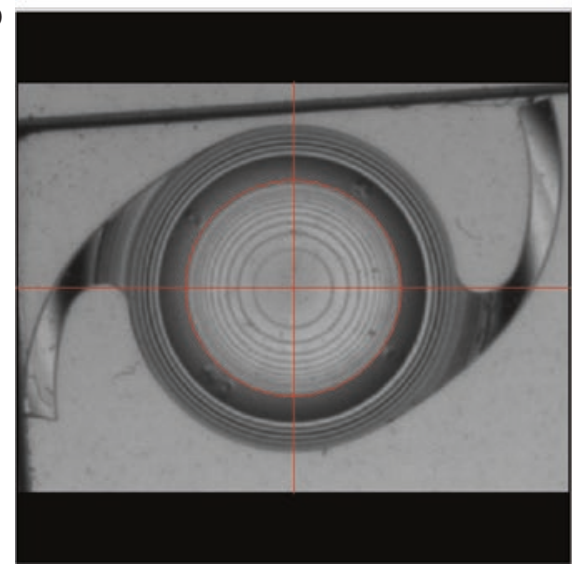

Optons | Deviason Maps |Modvision Mas | Poner Maps |Zernike MTF

\begin{tabular}{l|l}
\hline Aperture: $3.00 \mathrm{~mm}$ & MTF[100]: 0.53 \\
Sphere: $5.05 \quad$ Cyl: 0.06 & MTF[50]: 0.75 \\
\hline & MTF[25]: 0.88
\end{tabular}

Strehl Rat. X: 0.967 Strehl Rat. Y: 0.968 Sph Aber: -0.0110

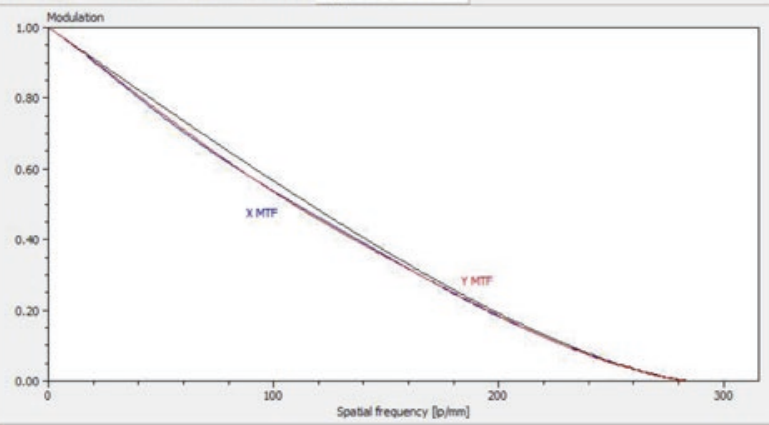

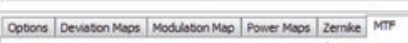

Aperture: $3.00 \mathrm{~mm}$

Sphere: $2.91 \quad$ Cyl: 0.08

Strehl Rat X: 0.346 Strehl Rat. Y: 0.387 Sph Aber

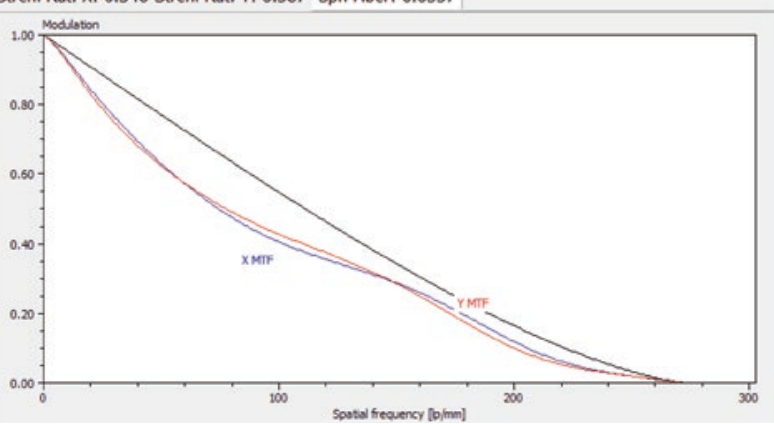

Fig. 15.9 Creation of a -2D RIS change inside one IOL. Diopter readings and MTF before (a) and after (b) RIS treatment 

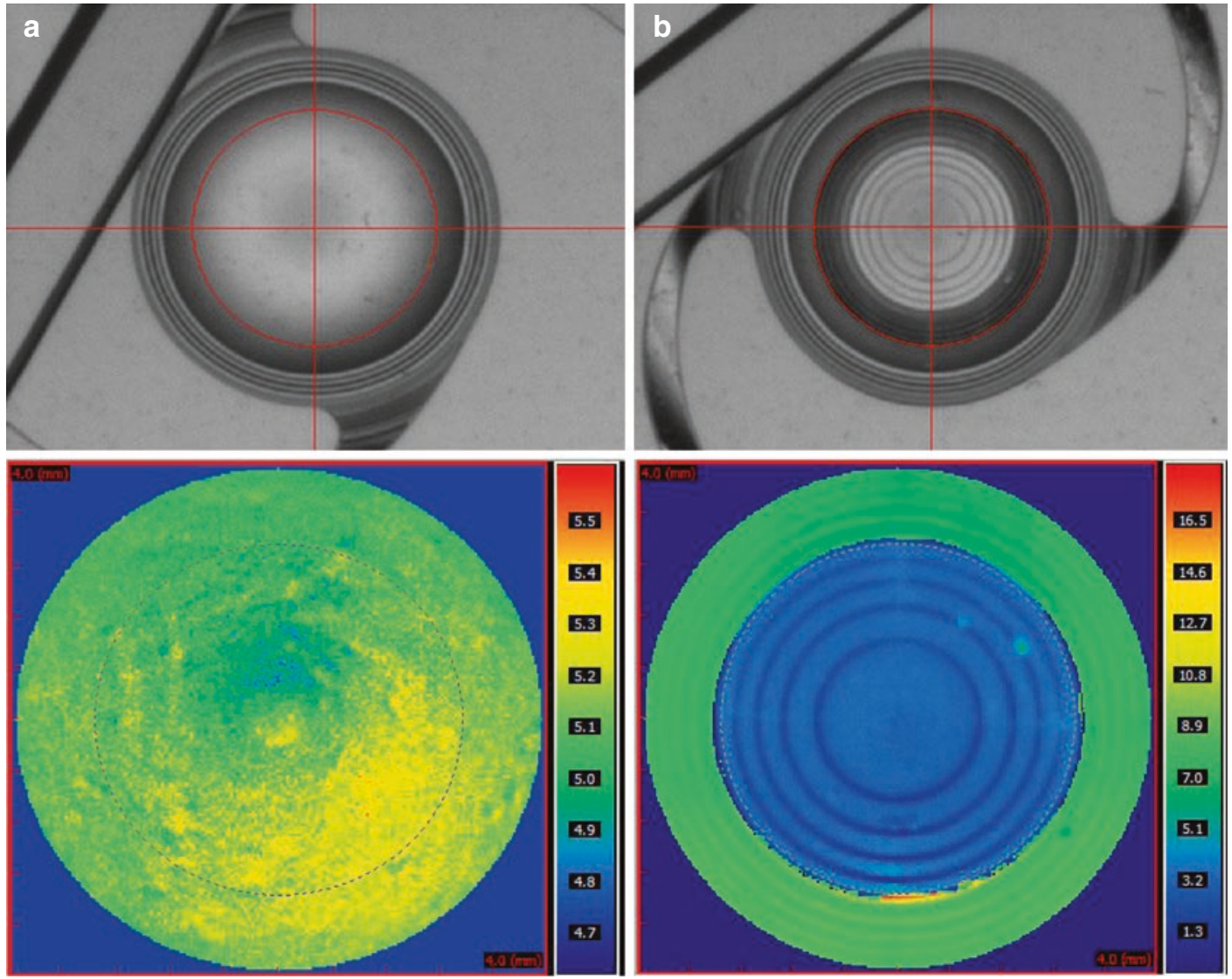

Fig. 15.10 Creation of a $-2 \mathrm{D}$ and $+2 \mathrm{D}$ RIS change inside one IOL. Modulation map and diopter power map readings before (a) and after (b) RIS treatment

since then to keep the final MTF on a minimum of 0.43 for spherical changes.

In Fig. 15.10, one of the original proofs of concept lenses is displayed. The top shows the original modulation map and the bottom the diopter power map measured using the Nimo from Lambda X. The original IOL measured $5 \mathrm{D}$ and the outside area was treated to have a $+2 \mathrm{D}$ change while the inside area had a $-2 \mathrm{D}$ RIS change resulting in a refractive multifocal IOL [31]. The shaping algorithm was further improved since to allow for a more precise shaping process, higher diopters and also diffractive multifocal lens shaping.

The consistency and precision of the power changes induced by the laser have been shown to be within $0.1 \mathrm{D}$ of the targeted change with- out a significant reduction in the MTF. As shown in Fig. 15.11, the same $-2.0 \mathrm{D}$ refractive index shaping lens was shaped into nine IOLs to assess the repeatability of the process [13]. Figure 15.11 shows the diopter change of the IOL after the shaping process.

\subsubsection{Adjustment of Sphere}

In Fig. 15.12, the creation of a refractive $+4 \mathrm{D}$ RIS lens is depicted. The original IOL measured 16.59 D with an MTF of 0.5 for $100 \mathrm{lp} / \mathrm{mm}$, after RIS the IOL measured 20.59 D with an MTF of $0.49 \mathrm{lp} / \mathrm{mm}$ [32]. Thus, the RIS technology can be used to change an existing IOL diopter of up to $4 \mathrm{D}$ while keeping a good MTF. 
Fig. 15.11 Repeatability of a -2 D refractive index shaping lens

\section{Repeatability}

(-2D RIS lens in IOL)

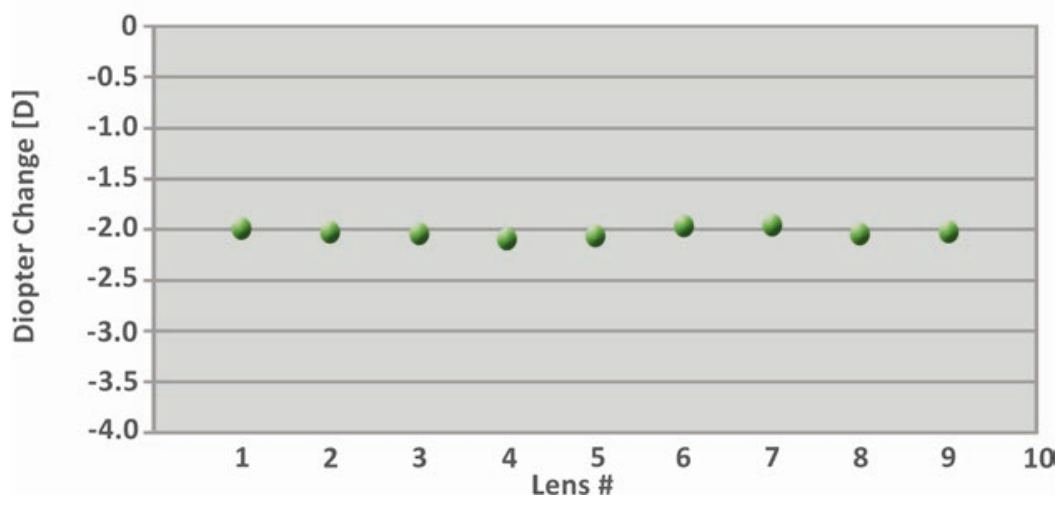

a

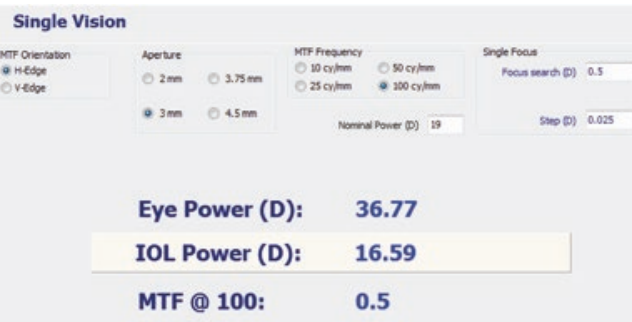

b

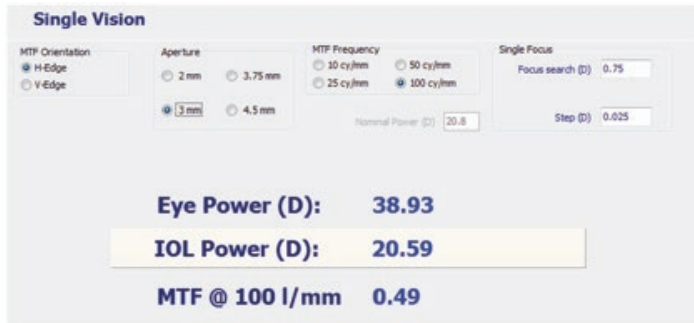

Fig. 15.12 Diopter readings and MTF before (a) and after (b) RIS treatment

\subsubsection{Conversion from Monofocal to a Toric IOL}

The RIS procedure is especially beneficial when it comes to the creation of toricity, the lens has already settled and the toric adjustment will therefore be centered and the axis is fixed. Figure 15.13 shows the creation of a toric lens, the original monofocal IOL measures $22 \mathrm{D}$ and after RIS a $3 \mathrm{D}$ astigmatism correction in one axis can be measured [33].

\subsubsection{Conversion from Monofocal to Multifocal}

In Fig. 15.14, the creation of multifocality in a monofocal hydrophobic IOL, is shown. Before treatment, the IOL power was $25.82 \mathrm{D}$, with an
MTF of 0.54 for $100 \mathrm{lp} / \mathrm{mm}$. After treatment, the IOL measures two foci, the original lens diopter and an additional 3.1 D add with a 62/38 split. Thus, the RIS technology can be used to add multifocality to a monofocal IOL.

\subsection{Biocompatibility of Intraocular Lens Power Adjustment}

An in vivo study on rabbit eyes confirmed that postoperative outcomes in terms of uveal and capsular biocompatibility were similar for treated lenses and untreated lenses. The laser power adjustment procedure did not induce inflammatory reactions in the eye or damage to the IOL optic.

Overall, all implantation procedures were uneventful and the IOLs could be fully injected 


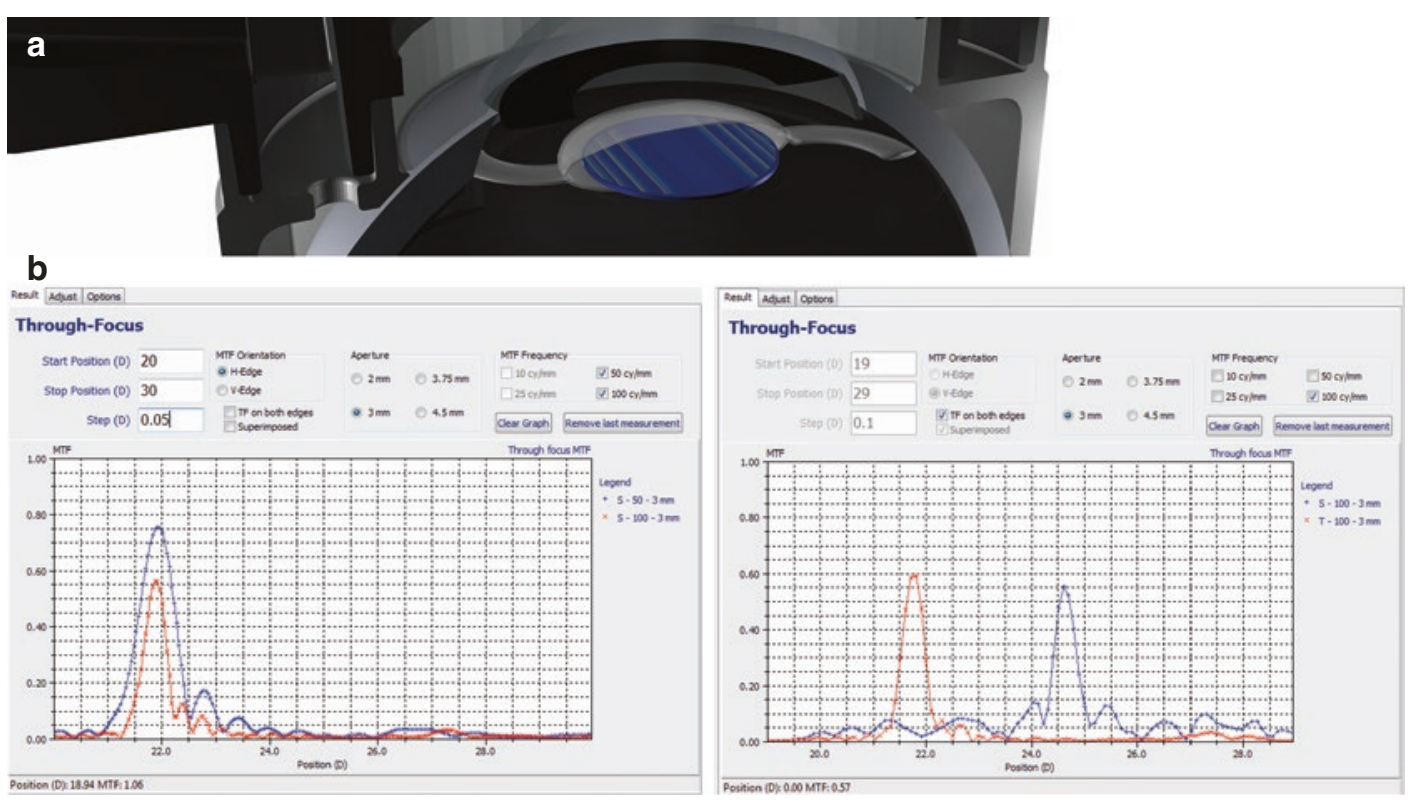

Fig. 15.13 Converting monofocal IOL into a toric IOL; schematic view (a), before and after RIS (b)

a

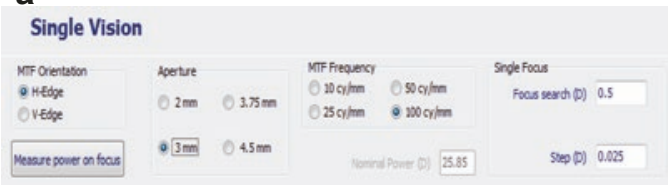

\begin{tabular}{ll} 
Eye Power (D): & 42.35 \\
IOL Power (D): & 25.82 \\
\hline MTF @ 100: & 0.54 \\
Strehl Ratio: & 0.79 \\
\hline
\end{tabular}

b

Multi-focal

\begin{tabular}{|c|c|c|c|c|c|c|}
\hline Star Postion (D) & 25 & \multirow{2}{*}{ 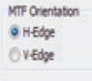 } & \multicolumn{2}{|l|}{ neetre } & \multicolumn{2}{|l|}{ Mif Freavecy } \\
\hline Susp Postion (D) & 29.5 & & $02 \mathrm{~m}$ & $037 \mathrm{sm}$ & $\begin{array}{l}0.00 \mathrm{~mm} \\
025 \mathrm{~mm}\end{array}$ & $\begin{array}{l}0.50 \mathrm{chmm} \\
0.100 \mathrm{rmm}\end{array}$ \\
\hline $\operatorname{Step}(D)$ & 0.05 & & $93 \mathrm{~m}$ & $045 \mathrm{~m}$ & & unber $\overline{3}$ \\
\hline
\end{tabular}

\begin{tabular}{lcc|c|} 
& Focus 1 & & Focus 2 \\
Eye Power (D): & 41.66 & & 43.36 \\
IOL Power (D): & 25.72 & & 28.82 \\
\hline Add (D): & & 3.1 \\
\hline MTF @ 100: & 0.37 & 0.21 \\
\hline Energy & 0.62 & 0.38 \\
\hline
\end{tabular}

Fig. 15.14 Conversion of a monofocal IOL to multifocal IOL, before (a) and after (b) RIS

within the capsular bag. At the 1-week examination, nearly all operated eyes had a mild inflammatory reaction with fibrin in front of the lens or at the level of the capsulorhexis edge. Fibrin formation had completely resolved by the 2 -week examination, when a mild amount of PCO started to be observed in nearly all eyes. Most eyes at this time point also had proliferative lens cortical material or pearl formation in front of the IOL.
All laser power adjustment procedures were also uneventful, and the duration of the laser treatment per se was fast (23 s). Under slit lamp examination, the phase-wrapped structure created by the laser could be observed within the optic substance of all treated IOLs. The examination also showed the formation of gas bubbles between the posterior surface of the IOL and the posterior capsule, which disappeared within 5 h. Other observa- 
tions included mild corneal edema and conjunctival infection, which could be related to the eye remaining open during the alignment step of the procedure. No aqueous flare, cells, iris hyperemia, or fibrin formations were observed at any of the post-laser slit lamp examinations, and the process did not create glistening's in the IOLs [34, 35].

The consistency and precision of the power changes induced by the laser have been shown in vitro. Another recent study [13] found that the refractive-index change altered the dioptric power of commercially available yellow hydrophobic acrylic IOLs to within $\pm 0.1 \mathrm{D}$ of the targeted change without a significant reduction in the MTF. A more recent study performed in our laboratory also showed the consistency and precision of the power change by this technology in commercially available hydrophobic acrylic lenses with and without a blue-light filter, without inducing significant changes in IOL light transmission.

Our current in vivo study confirmed that postoperative outcomes in terms of uveal and capsular biocompatibility were similar between treated lenses and untreated lenses, as shown during clinical examination and by complete histopathology. The laser power adjustment procedure did not induce inflammatory reactions in the eye or damage to the IOL optic. Alignment of the rabbit eye under the laser system for the adjustment procedure was challenging because it was necessary to anesthetize the animal, which would not be the case in a clinical situation. Even though an eye interface had to be specially designed for this study, which was also the first performed in vivo, the change in power obtained was consistent in the group of treated eyes. It is noteworthy that power measurements of the IOLs were not performed before implantation in the rabbit eyes to avoid compromising the sterility of the IOLs because the main objective of the current study was to evaluate biocompatibility after laser treatment. Therefore, the method used to estimate the changes in power after laser treatment was based on measurements done with the power and MTF device after IOL explantation (Table 15.2).

The most likely cause of postoperative refractive errors after IOL implantation is incorrect
Table 15.2 Power of the IOLs implanted in the rabbit eyes, measured with a PMTF device after explantation of the lenses 4 weeks postoperatively after full hydration

\begin{tabular}{l|l|l|l}
\hline & IOL power (D) & & \\
\hline Rabbit & Treated & $\begin{array}{l}\text { Contralateral } \\
\text { untreated }\end{array}$ & Change \\
\hline 1 & +26.5 & +23.2 & +3.3 \\
\hline 2 & +26.9 & +23.2 & +3.7 \\
\hline 3 & +27.0 & +23.7 & +3.3 \\
\hline 4 & +26.7 & +23.1 & +3.6 \\
\hline 5 & +27.0 & +23.0 & +4.0 \\
\hline 6 & +26.8 & +23.2 & +3.6 \\
\hline
\end{tabular}

IOL intraocular lens

IOL calculation resulting from incorrect measurements of the eye [36]. Also, current standards regarding IOL power labeling allow a tolerance of $\pm 0.30 \mathrm{D}$ for IOLs of $0.00 \mathrm{D}$ to $15.00 \mathrm{D}$ or less. The tolerance increases to $\pm 0.40 \mathrm{D}$ for IOLs with a power from greater than $15.00 \mathrm{D}$ to $25.00 \mathrm{D}$ or less, which means that an IOL of $22.61 \mathrm{D}$ and another of $23.39 \mathrm{D}$ could be labeled with a dioptric power of 23.00 D or the IOL of 23.39 D could be labeled as both 23.0 D and 23.5 D [37]. All these factors make postoperative IOL adjustment technologies particularly interesting.

\subsection{Discussion and Conclusion}

The RIS treatment (see e.g. Fig. 15.1a) uses a femtosecond laser to change the hydrophilicity of the targeted area, which allows for a change in the refractive index. This effect in combination with a two dimensional scan pattern allows for the creation of a refractive or diffractive lens inside the material.

A photochemical process was identified, wherein hydrophilic polar functional groups are generated by photo-induced hydrolysis of polymeric material, in areas which are exposed to a femtosecond laser, thus providing the chemical basis for a hydrophilicity based refractive index change, facilitating the creation of a RIS-lens. The newly formed functional groups, e.g. amines and carboxylic acids, are strongly hydrophilic. The molecules are monomers or dimers, embedded in the original polymer and the UV-absorber co-polymer. These molecules remain in their 
existing place and are modified by the exposure to the laser light. In three different polymeric materials, fluorophores with identical spectral signatures were detected. Thus, photo-induced hydrolysis results in rearrangements of chemical bonds, essentially within the UV-absorber molecule, preserving the integrity of the polymeric material. Based on fluorescence microscopy, STED microscopy and Raman microscopy, no leachables are generated. Also, standard leachable tests have been performed on RIS-modified IOLs, and no leachables were found.

The results of the first in vivo study evaluating the biocompatibility of this new application of the femtosecond laser are reported. Refractive Index Shaping (RIS) can be applied to any commercially available hydrophobic or hydrophilic acrylic IOL because the process does not depend on a special IOL material. Power adjustment is noninvasive and fast and can be performed under topical anesthesia. The dioptric power of the IOL can be increased or decreased to account for surgical errors, IOL tilt, IOL decentration, or a change in the physical characteristics of the eye. Multiple adjustments to the same IOL can be performed because each adjustment only changes a very thin layer within the IOL optic substance. Premium functions can be added to the IOL and removed later, if necessary. An added multifocal pattern can, for example, be canceled by application of a pattern with opposite characteristics. The use of special protective spectacles is not necessary after treatment and the process works with standard hydrophilic and hydrophobic available intraocular lenses.

Refractive Index Shaping (RIS) is an exciting technology with the ability to precisely change the power of an intraocular lens. The RIS process is not based on a lens but on a device, which is currently not yet approved. This technology has the potential to change the course of ophthalmic cataract surgery and lens accuracy in the future. It is hopeful that this technology will allow a minimally invasive treatment for the management of refractive surprises after cataract surgery. It is exciting to imagine treatments to improve residual refractive errors will minimally invasive office procedure. To remove the surgical risks and move the treatment from the operating room to an in-office procedure.

In conclusion, postoperative lens customization utilizes femtosecond laser technology to adjust the power of an implanted IOL. A minimally invasive laser treatment provides a customized vision correction of a patient, who has had previous cataract surgery, to optimize the patient's vision. This new technology gives the surgeon an additional opportunity to improve a patient's sight. It separates the customization of the lens from the original cataract surgery giving both the patient and the doctor time to discuss and consider this treatment.

\section{References}

1. Daily S. What is holding back the premium IOL channel's growth? CRSToday. 2012. https://crstoday.com/ articles/2012-jan/what-is-holding-back-the-premiumiol-channels-growth. Accessed 18 Oct 2018.

2. Hengerer FH, Conrad-Hengerer I, Buchner SE, Dick HB. Evaluation of the calhoun vision UV light adjustable lens implanted following cataract removal. J Refract Surg. 2010;26(10):716-21.

3. Hill W. Distribution of corneal astigmatism-normal adult population. https://www.doctor-hill.com/iolmain/astigmatism_chart.htm. Accessed 6 Mar 2019.

4. Villegas EA, Alcon E, Artal P. Minimum amount of astigmatism that should be corrected. J Cataract Refract Surg. 2014;40(1):13-9. https://doi.org/10.1016/j. jcrs.2013.09.010.

5. Manning S, Barry P, Henry Y, et al. Femtosecond laserassisted cataract surgery versus standard phacoemulsification cataract surgery: Study from the European Registry of Quality Outcomes for Cataract and Refractive Surgery. J Cataract Refract Surg. 2016;42(12):1779-90. https://doi.org/10.1016/j.jcrs.2016.10.013.

6. Ohmachi Y, Igo T. Laser-induced refractive-index change in As-S-Ge glasses. Appl Phys Lett. 1972;20(12):506-8.

7. Ding L, Blackwell R, Kunzler JF, Knox H. Large refractive index change in silicone-based and nonsilicone-based hydrogel polymers induced by femtosecond laser micro-machining. Opt Express. 2006;14(24):11901-9.

8. Ding L. Micro-processing of polymers and biological materials using high repetition rate femtosecond laser pulses. Dissertation, University of Rochester. 2009.

9. Takeshima N, Kuroiwa Y, Narita Y, Tanaka S, Hirao K. Fabrication of a periodic structure with a high refractive-index difference by femtosecond laser pulses. Opt Express. 2004;12(17):4019-24. 
10. Katayama S, Horiike M. Plastic object. US Patent 2002/0117624A1, 29 Aug 2002. 2002.

11. Sahler R, Zhou SQ, Bille JF. Hydrophilicity alteration system and method. US Patent 9186242 B2, 17 Nov 2015. 2015.

12. Bille JF, Engelhardt J, Volpp H, Laghouissa A, Motzkus M, Jiang Z, Sahler R. Chemical basis for alteration of an intraocular lens using femtosecond laser. Biomed Opt Exp. 2017;8(3):1390-404.

13. Sahler R, Bille JF, Enright S, Chhoeung S, Chan $\mathrm{K}$. Creation of a refractive lens within an existing intraocular lens using a femtosecond laser. J Cataract Refract Surg. 2016;42(8):1207-15.

14. Sahler R, Bille JF, Schanzlin D. In vivo IOL modification. MillennialEye. 2016. https://millennialeye. com/articles/2016-nov-dec/in-vivo-iol-modification. Accessed 18 Oct 2018.

15. Bracco G, Holst B. Surface science techniques in springer series in surface sciences. Berlin, Heidelberg: Springer; 2013.

16. Görlitz F, Hoyer P, Falk H, Kastrup L, Engelhardt J, Hell SW. A STED microscope designed for routine biomedical applications. Prog Electromagn Res. 2014;147:57-68.

17. Technical Manual, Xplo RATM Plus System. http:// www.horiba.com/fileadmin/uploads/Scientific/ Documents/Raman/Brochure_XploRA_Series062016-B.pdf. Accessed 18 Oct 2018.

18. Carl Zeiss Meditec, Jena. "Hydrophilic IOLs. http://www.zeiss.ph/C 125679 E00525939/ EmbedTitelIntern/hydrophil_acryl_hapticdesign_ eng/\$File/hydrophil_acryl_hapticdesign_eng.pdf.

19. Carl Zeiss Meditec, Jena. Hydrophobic IOLs, https:// www.zeiss.com/meditec/int/products/ophthalmology-optometry/cataract/iol-implantation/hydrophobic-c-loop-iols/ct-lucia.html\#technical-data. Accessed 18 Oct 2018.

20. AMO, Santa Ana. Hydrophobic IOLs, https://www. abbottmedicaloptics.com/pdf/TECNIS_One_Piece_ Insert.pdf.

21. Wang ZK, Zheng HY, Lim CP, Lam YC. Polymer hydrophilicity and hydrophobicity induced by femtosecond laser direct irradiation. Appl Phys Lett. 2009;95(11):111110.

22. Wochnowski C, Shams Eldin MA, Metev S. UV-laserassisted degradation of poly (methyl methacrylate). Polym Degrad Stabil. 2005;89(2):252-64.

23. Sun HB, Kawata S. Two-photon photopolymerization and 3D Lithographic microfabrication. Adv Polym Sci. 2004;170:169-273.

24. Zhou WH, Kuebler SM, Brau KL, Yu TY, Cammack JK, Ober CK, Perry JW, Marder SR. An efficient twophoton generated photoacid applied to positive tone 3D microfabrication. Science. 2002;296:1106-9.
25. Kemal E, Deb S. Design and synthesis of threedimensional hydrogel scaffolds for intervertebral disc repair. J Mater Chem. 2012;22(21):10725-34.

26. Perova TS, Vij JK, Xu H. Fourier transform infrared study of poly (2-hydroxyethyl methacrylate) PHEMA. Colloid Polym Sci. 1997;275(4):323-32.

27. Bertoluzza A, Monti P, Garcia-Ramos JV, Simoni R, Caramazza R, Calzavara A. Applications of Raman spectroscopy to the ophthalmological field: Raman spectra of soft contact lenses made of poly2-hydroxyethylmethacrylate (PHEMA). J Mol Struct. 1986;143(1-2):469-72.

28. Werner T. Triplet deactivation in benzotriazole-type ultraviolet stabilizers. J Phys Chem. 1979;83(3):320-5.

29. Miladinova PM, Konstantinova TN. Photostabilizers for polymers - new trends. J Chem. Technol. Metall. 2015;50(3):229-39.

30. Mabilleau G, Cincu C, Baslé MF, Chappard D. Polymerization of 2-(hydroxyethyl) methacrylate by two different initiator/accelerator systems: a Raman spectroscopic monitoring. J Raman Spectrosc. 2008;39(7):767-71.

31. Sahler R, Bille JF, Enright S, Chhoeung S, Chan K. Customizable IOL: full-sized lens created inside existing IOL using laser induced refractive index change. ASCRS. 2018. https://ascrs.confex.com/ ascrs/15am/webprogram/Paper15512.html. Accessed 18 Oct 2018.

32. Sahler R, Enright S, Chhoeung S, Chan K, Bille JB, Alley RK. Progressive soaking process of the refractive index-shaped lens.. ASCRS. 2018. https:// ascrs.confex.com/ascrs/18am/meetingapp.cgi/ Paper/46404. Accessed 18 Oct 2018.

33. Sahler R, Bille JF, Enright $\mathrm{S}$ et al. Simulaneous refractive and toric creation insdie a standard hydrophobic intraocular lens using a femtosecond laser. ESCRS. 2017. http://www.escrs.org/Lisbon2017/programme/ free-papers-details.asp id $=28128 \&$ day $=0$. Accessed 18 Oct 2018.

34. Werner L, Ludlov J, Nguyen J, et al. Biocompatibility of intraocular lens power adjustment using a femtosecond laser in a rabbit model. J Cataract Refract Surg. 2017;43(8):1100-6.

35. Nguyen J, Liliana W, Ludlow J, Aliancy J, Ha L, Masino B, Enright S, Alley SK, Sahler R. Intraocular lens power adjustment by a femtosecond laser. J Cataract Refract Surg. 2018;44(2):226-30.

36. Ford J, Werner L, Mamalis N. Adjustable intraocular lens power technology. J Cataract Refract Surg. 2014;40:1205-23.

37. Hoffer KJ, Calogero D, Faaland RW, Ilev IK. Testing the dioptric power accuracy of exact-powerlabeled intraocular lenses. J Cataract Refract Surg. 2009;35:1995-9. 
Open Access This chapter is licensed under the terms of the Creative Commons Attribution 4.0 International License (http://creativecommons.org/licenses/by/4.0/), which permits use, sharing, adaptation, distribution and reproduction in any medium or format, as long as you give appropriate credit to the original author(s) and the source, provide a link to the Creative Commons license and indicate if changes were made.

The images or other third party material in this chapter are included in the chapter's Creative Commons license, unless indicated otherwise in a credit line to the material. If material is not included in the chapter's Creative Commons license and your intended use is not permitted by statutory regulation or exceeds the permitted use, you will need to obtain permission directly from the copyright holder.

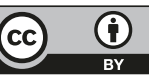

